Eng. Appl. Sci. Lett., Vol. 1(2018), No. 1, pp. 10 - 15

Website: https://pisrt.org/psr-press/journals/easl/

ISSN: 2617-9709 (Online) 2617-9695 (Print)

http://dx.doi.org/10.30538/psrp-easl2018.0002

\title{
COMPLETE MONOTONICITY PROPERTIES OF A FUNCTION INVOLVING THE POLYGAMMA FUNCTION
}

\author{
KWARA NANTOMAH ${ }^{1}$
}

ABSTRACT. In this paper, we study completete monotonicity properties of certain functions associated with the polygamma functions. Subsequently, we deduce some inequalities involving difference of polygamma functions.

Index Terms: Polygamma function; complete monotonicity; inequality.

\section{Introduction}

The classical Gamma function, which is an extension of the factorial notation to noninteger values is usually defined as

$$
\Gamma(x)=\int_{0}^{\infty} t^{x-1} e^{-t} d t, \quad x>0,
$$

and satisfying the basic property

$$
\Gamma(x+1)=x \Gamma(x), \quad x>0 .
$$

Its logarithmic derivative, which is called the Psi or digamma function is defined as (see [1] and [2])

$$
\begin{aligned}
\psi(x)=\frac{d}{d x} \ln \Gamma(x) & =-\gamma+\int_{0}^{\infty} \frac{e^{-t}-e^{-x t}}{1-e^{-t}} d t, & & x>0, \\
& =-\gamma-\frac{1}{x}+\sum_{k=1}^{\infty} \frac{x}{k(k+x)}, \quad & & x>0,
\end{aligned}
$$

Received 25-09-2018. Revised 27-10-2018. Accepted 29-10-2018.

${ }^{1}$ Corresponding Author

(C) 2018 Kwara Nantomah. This is an open access article distributed under the Creative Commons Attribution License, which permits unrestricted use, distribution, and reproduction in any medium, provided the original work is properly cited. 
where $\gamma=\lim _{n \rightarrow \infty}\left(\sum_{k=1}^{n} \frac{1}{k}-\ln n\right)=0.577215664 \ldots$ is the Euler-Mascheroni's constant. Derivatives of the Psi function, which are called polygamma functions are given as [1]

$$
\begin{aligned}
\psi^{(n)}(x) & =(-1)^{n+1} \int_{0}^{\infty} \frac{t^{n} e^{-x t}}{1-e^{-t}} d t, \quad x>0, \\
& =(-1)^{n+1} n ! \sum_{k=0}^{\infty} \frac{1}{(k+x)^{n+1}}, \quad x>0,
\end{aligned}
$$

satisfying the functional equation [1]

$$
\psi^{(n)}(x+1)=\psi^{(n)}(x)+\frac{(-1)^{n} n !}{x^{n+1}}, \quad x>0,
$$

where $n \in \mathbb{N}_{0}$ and $\psi^{(0)}(x) \equiv \psi(x)$. Here, and for the rest of this paper, we use the notations: $\mathbb{N}=\{1,2,3,4, \ldots\}, \mathbb{N}_{0}=\mathbb{N} \cup\{0\}$ and $\mathbb{R}=(-\infty, \infty)$. Also, it is well known in the literature that the integral

$$
\frac{n !}{x^{n+1}}=\int_{0}^{\infty} t^{n} e^{-x t} d t
$$

holds for $x>0$ and $n \in \mathbb{N}_{0}$. See for instance [1]. In [3], Qiu and Vuorinen established among other things that the function

$$
h_{1}=\psi\left(x+\frac{1}{2}\right)-\psi(x)-\frac{1}{2 x},
$$

is strictly decreasing and convex on $(0, \infty)$. Motivated by this result, Mortici [4] proved a more generalized and deeper result which states that, the function

$$
f_{a}=\psi(x+a)-\psi(x)-\frac{a}{x}, \quad a \in(0,1),
$$

is strictly completely monotonic on $(0, \infty)$. Recall that a function $f:(0, \infty) \rightarrow \mathbb{R}$ is said to be completely monotonic on $(0, \infty)$ if $f$ has derivatives of all order and $(-1)^{n} f^{(n)}(x) \geq 0$ for all $x \in(0, \infty)$ and $n \in \mathbb{N}_{0}$.

In this paper, the objective is to extend Mortici's results to the polygamma functions.

\section{Some Lemmas}

In order to establish our main results, we need the following lemmas.

Lemma 2.1. Let a function $q_{\alpha, \beta}(t)$ be defined as

$$
q_{\alpha, \beta}(t)= \begin{cases}\frac{e^{-\alpha t}-e^{-\beta t}}{1-e^{-t}}, & t \neq 0, \\ \beta-\alpha, & t=0,\end{cases}
$$

where $\alpha, \beta$ are real numbers such that $\alpha \neq \beta$ and $(\alpha, \beta) \notin\{(0,1),(1,0)\}$. Then $q_{\alpha, \beta}(t)$ is increasing on $(0, \infty)$ if and only if $(\beta-\alpha)(1-\alpha-\beta) \geq 0$ and $(\beta-$ $\alpha)(|\alpha-\beta|-\alpha-\beta) \geq 0$.

Proof. See [5, 6] or 7]. 
Lemma 2.2. Let $a \in(0,1)$. Then the inequality

$$
a<\frac{1-e^{-a t}}{1-e^{-t}}<1
$$

holds for $t \in(0, \infty)$.

Proof. Note that the function $h(t)=\frac{1-e^{-a t}}{1-e^{-t}}$ which is obtained from Lemma 2.1 by letting $\alpha=0$ and $\beta=a \in(0,1)$ is increasing on $(0, \infty)$. Also,

$$
\lim _{t \rightarrow 0^{+}} h(t)=a \text { and } \quad \lim _{t \rightarrow \infty} h(t)=1 .
$$

Then for $t \in(0, \infty)$, we have

$$
a=\lim _{t \rightarrow 0^{+}} h(t)=h(0)<h(t)<h(\infty)=\lim _{t \rightarrow \infty} h(t)=1,
$$

which gives inequality (8).

\section{Main Results}

We now present our results in this section.

Theorem 3.1. Let $f_{a, k}(x)$ and $h_{a, r}(x)$ be defined for $a \in(0,1), k \in\{2 s: s \in$ $\left.\mathbb{N}_{0}\right\}, r \in\left\{2 s+1: s \in \mathbb{N}_{0}\right\}$ and $x \in(0, \infty)$ as

$$
f_{a, k}(x)=\psi^{(k)}(x+a)-\psi^{(k)}(x)-\frac{a k !}{x^{k+1}},
$$

and

$$
h_{a, r}(x)=\psi^{(r)}(x+a)-\psi^{(r)}(x)-\frac{a r !}{x^{r+1}} .
$$

Then $f_{a, k}(x)$ and $-h_{a, r}(x)$ are strictly completely monotonic on $(0, \infty)$.

Proof. By repeated differentiations with respect to $x$, and by using (2) and (4), we obtain

$$
\begin{aligned}
f_{a, k}^{(n)}(x)= & \psi^{(k+n)}(x+a)-\psi^{(k+n)}(x)-\frac{(-1)^{n} a(k+n) !}{x^{k+n+1}} \\
= & (-1)^{k+n+1} \int_{0}^{\infty} \frac{t^{k+n} e^{-(x+a) t}}{1-e^{-t}} d t-(-1)^{k+n+1} \int_{0}^{\infty} \frac{t^{k+n} e^{-x t}}{1-e^{-t}} d t \\
& -(-1)^{n} a \int_{0}^{\infty} t^{k+n} e^{-x t} d t .
\end{aligned}
$$

This implies that

$$
\begin{aligned}
(-1)^{n} f_{a, k}^{(n)}(x) & =-\int_{0}^{\infty} \frac{t^{k+n} e^{-x t} e^{-a t}}{1-e^{-t}} d t+\int_{0}^{\infty} \frac{t^{k+n} e^{-x t}}{1-e^{-t}} d t-a \int_{0}^{\infty} t^{k+n} e^{-x t} d t \\
& =\int_{0}^{\infty}\left[\frac{1-e^{-a t}}{1-e^{-t}}-a\right] t^{k+n} e^{-x t} d t \\
& >0
\end{aligned}
$$


which is as a result of Lemma 2.2. Alternatively, we could proceed as follows.

$$
\begin{aligned}
(-1)^{n} f_{a, k}^{(n)}(x) & =\int_{0}^{\infty}\left[\frac{1-e^{-a t}}{1-e^{-t}}-a\right] t^{k+n} e^{-x t} d t \\
& =a \int_{0}^{\infty}\left[\frac{1-e^{-a t}}{a t}-\frac{1-e^{-t}}{t}\right] \frac{t^{k+n+1} e^{-x t}}{1-e^{-t}} d t \\
& >0 .
\end{aligned}
$$

Notice that, since the function $\frac{1-e^{-t}}{t}$ is strictly decreasing on $(0, \infty)$, then for $a \in$ $(0,1)$, we have $\frac{1-e^{-a t}}{a t}>\frac{1-e^{-t}}{t}$. Hence $f_{a, k}(x)$ is strictly completely monotonic on $(0, \infty)$. Similarly, we have

$$
\begin{aligned}
-h_{a, r}^{(n)}(x)= & \frac{(-1)^{n} a(r+n) !}{x^{r+n+1}}+\psi^{(r+n)}(x)-\psi^{(r+n)}(x+a) \\
= & (-1)^{n} a \int_{0}^{\infty} t^{r+n} e^{-x t} d t+(-1)^{r+n+1} \int_{0}^{\infty} \frac{t^{r+n} e^{-x t}}{1-e^{-t}} d t \\
& -(-1)^{r+n+1} \int_{0}^{\infty} \frac{t^{r+n} e^{-(x+a) t}}{1-e^{-t}} d t,
\end{aligned}
$$

which implies that

$$
\begin{aligned}
(-1)^{n}\left(-h_{a, r}\right)^{(n)}(x) & =a \int_{0}^{\infty} t^{r+n} e^{-x t} d t+\int_{0}^{\infty} \frac{t^{r+n} e^{-x t}}{1-e^{-t}} d t-\int_{0}^{\infty} \frac{t^{r+n} e^{-x t} e^{-a t}}{1-e^{-t}} d t \\
& =\int_{0}^{\infty}\left[a+\frac{1-e^{-a t}}{1-e^{-t}}\right] t^{r+n} e^{-x t} d t \\
& >0 .
\end{aligned}
$$

Hence $-h_{a, r}(x)$ is strictly completely monotonic on $(0, \infty)$.

Remark 3.2. Since every completely monotonic function is convex and decreasing, it follows that $f_{a, k}(x)$ is strictly convex and strictly decreasing on $(0, \infty)$. In this way, $h_{a, r}(x)$ is strictly concave and strictly increasing on $(0, \infty)$.

Corollary 3.3. The inequality

$$
\frac{a k !}{x^{k+1}}<\psi^{(k)}(x+a)-\psi^{(k)}(x)<\psi^{(k)}(a)-\psi^{(k)}(1)+k !\left(\frac{a}{x^{k+1}}+\frac{1}{a^{k+1}}-a\right),
$$

holds for $a \in(0,1), k \in\left\{2 s: s \in \mathbb{N}_{0}\right\}$ and $x \in(1, \infty)$.

Proof. Since $f_{a, k}(x)$ is decreasing, then for $x \in(1, \infty)$ and by applying (3), we obtain

$$
\begin{aligned}
0=\lim _{x \rightarrow \infty} f_{a, k}(x)<f_{a, k}(x)<f_{a, k}(1) & =\psi^{(k)}(a+1)-\psi^{(k)}(1)-a k ! \\
& =\psi^{(k)}(a)-\psi^{(k)}(1)+\frac{k !}{a^{k+1}}-a k !,
\end{aligned}
$$

which completes the proof. 
Remark 3.4. In particular, if $a=\frac{1}{2}$ and $k=0$ in Corollary 3.3 , then we obtain

$$
\frac{1}{2 x}<\psi\left(x+\frac{1}{2}\right)-\psi(x)<\frac{1}{2 x}+\frac{3}{2}-2 \ln 2, \quad x \in(1, \infty) .
$$

Also, if $a=\frac{1}{2}$ and $k=2$ in Corollary 3.3 then we obtain

$$
\frac{1}{x^{3}}<\psi^{\prime \prime}\left(x+\frac{1}{2}\right)-\psi^{\prime \prime}(x)<\frac{1}{x^{3}}+15-12 \zeta(3), \quad x \in(1, \infty),
$$

where $\zeta(x)$ is the Riemann zeta function.

Corollary 3.5. The inequality

$$
\psi^{(r)}(a)-\psi^{(r)}(1)+r !\left(\frac{a}{x^{r+1}}-\frac{1}{a^{r+1}}-a\right)<\psi^{(r)}(x+a)-\psi^{(r)}(x)<\frac{a r !}{x^{r+1}},
$$

holds for $a \in(0,1), r \in\left\{2 s+1: s \in \mathbb{N}_{0}\right\}$ and $x \in(1, \infty)$.

Proof. Likewise, since $h_{a, r}(x)$ is increasing, then for $x \in(1, \infty)$, we obtain

$$
\psi^{(r)}(a)-\psi^{(r)}(1)-\frac{r !}{a^{r+1}}-a r !=h_{a, r}(1)<h_{a, r}(x)<\lim _{x \rightarrow \infty} h_{a, r}(x)=0,
$$

which yields (14).

Remark 3.6. If $a=\frac{1}{2}$ and $r=1$ in Corollary 3.5 then we obtain

$$
\frac{1}{2 x^{2}}+\frac{\pi^{2}}{3}-\frac{9}{2}<\psi^{\prime}\left(x+\frac{1}{2}\right)-\psi^{\prime}(x)<\frac{1}{2 x^{2}}, \quad x \in(1, \infty) .
$$

Furthermore, if $a=\frac{1}{2}$ and $r=3$ in Corollary 3.5 then we obtain

$$
\frac{3}{x^{4}}+\frac{14 \pi^{4}}{15}-99<\psi^{\prime \prime \prime}\left(x+\frac{1}{2}\right)-\psi^{\prime \prime \prime}(x)<\frac{3}{x^{4}}, \quad x \in(1, \infty)
$$

Remark 3.7. If $k=0$ in Theorem 3.1, then we obtain the main results of [4] as a special case of the present results.

Remark 3.8. This paper is a modified version of the preprint $[8]$.

\section{Competing Interests}

The author declares that there are no competing interests regarding the publication of this paper.

\section{REFERENCES}

1. Abramowitz, M., \& Stegun, I. A. (1972). Handbook of Mathematical Functions with Formulas, Graphs, and Mathematical Tables, National Bureau of Standards, Applied Mathematics Series 55, 10th Printing, Washington.

2. Olver, F. W., Lozier, D. W., Boisvert, R. F., \& Clark, C. W. (Eds.). (2010). NIST handbook of mathematical functions. Cambridge University Press, New York.

3. Qiu, S. L., \& Vuorinen, M. (2005). Some properties of the gamma and psi functions, with applications. Mathematics of computation, 74(250), 723-742. 
4. Mortici, C. (2010). A sharp inequality involving the psi function. Acta Universitatis Apulensis, 22, 41-45.

5. Guo, B. N., \& Qi, F. (2009). Properties and applications of a function involving exponential functions. Commun. Pure Appl. Anal, 8(4), 1231-1249.

6. Qi, F. (2010). Bounds for the ratio of two gamma functions. Journal of Inequalities and Applications, 2010(1), 493058.

7. Qi, F., \& Luo, Q. M. (2012). Bounds for the ratio of two gamma functions-From Wendel's and related inequalities to logarithmically completely monotonic functions. Banach Journal of Mathematical Analysis, 6(2), 132-158.

8. Nantomah, K. (2018). Complete monotonicity properties of a function involving the polygamma function. arXiv preprint arXiv:1807.05257.

\section{Kwara Nantomah}

Department of Mathematics, Faculty of Mathematical Sciences, University for Development Studies, Navrongo Campus, P. O. Box 24, Navrongo, UE/R, Ghana.

e-mail: knantomah@uds.edu.gh 